\begin{tabular}{|c|c|}
\hline Title & Characteristic polynomials of Linial arrangements for exceptional root systems \\
\hline Author(s) & Yoshinaga, Masahiko \\
\hline Citation & $\begin{array}{l}\text { Journal of combinatorial theory. Series A, 157, 267-286 } \\
\text { https://doi.org/10.1016/.jcta.2018.02.011 }\end{array}$ \\
\hline Issue Date & $2018-07$ \\
\hline Doc URL & http:/hdl.handle.net/2115/79003 \\
\hline Rights & $\begin{array}{l}\text { (2) 2018. Ins manuscript version is made avallable under the CC-BY-IVC-NVD } 4.0 \text { IIcense } \\
\text { http://reativecommons.org/icenses/by-nc-n/4.0/ }\end{array}$ \\
\hline Rights(URL) & https://creativecommons.org/icenses/by-nc-nd/4.0/ \\
\hline Type & article (author version) \\
\hline File Information & zeros03.pdf \\
\hline
\end{tabular}

Instructions for use 


\title{
Characteristic polynomials of Linial arrangements for exceptional root systems
}

\author{
Masahiko Yoshinaga*
}

May 11, 2017

\begin{abstract}
The (extended) Linial arrangement $\mathcal{L}_{\Phi}^{m}$ is a certain finite truncation of the affine Weyl arrangement of a root system $\Phi$ with a parameter $m$. Postnikov and Stanley conjectured that all roots of the characteristic polynomial of $\mathcal{L}_{\Phi}^{m}$ have the same real part, and this has been proved for the root systems of classical types.

In this paper we prove that the conjecture is true for exceptional root systems when the parameter $m$ is sufficiently large.

The proof is based on representations of the characteristic quasipolynomials in terms of Eulerian polynomials.
\end{abstract}

Keywords: Hyperplane arrangements, Linial arrangements, characteristic polynomials

\section{Contents}

1 Introduction $\quad 2$

1.1 Background ...................... 2

1.2 Main results . . . . . . . . . . . . . . . . . . 2

1.3 What makes roots lie on a line? . . . . . . . . . . . 3

2 Preliminaries $\quad 4$

2.1 Quasi-polynomials with the $G C D$-property . . . . . . . . . 4

2.2 Characteristic quasi-polynomials . . . . . . . . . . 5

2.3 Eulerian polynomials for root systems . . . . . . . . . . 6

2.4 Ehrhart quasi-polynomials for root systems . . . . . . . . . 8

2.5 Characteristic quasi-polynomials of Linial arrangements . . . . 9

${ }^{*}$ Department of Mathematics, Hokkaido University, North 10, West 8, Kita-ku, Sapporo 060-0810, JAPAN E-mail: yoshinaga@math.sci.hokudai.ac.jp 
3 Limit Polynomials $\quad 10$

3.1 Normalized limit polynomials . . . . . . . . . . . . . . . . . . 10

3.2 Truncated Eulerian polynomials . . . . . . . . . . . . 11

4 A Toy Case $r$

5 Main Results $\quad 14$

5.1 Settings . . . . . . . . . . . . . . . . . . 14

5.2 Asymptotic behavior of roots . . . . . . . . . . . 16

5.3 Exact arrangement of roots . . . . . . . . . . . . . 17

\section{Introduction}

\subsection{Background}

A hyperplane arrangement $\mathcal{A}=\left\{H_{1}, \ldots, H_{n}\right\}$ is a finite collection of affine hyperplanes in an $\ell$-dimensional vector space $\mathbb{K}^{\ell}$. Despite its simplicity, the theory of hyperplane arrangements has fruitful connections with many areas in mathematics $([20,23])$. One of the most important invariants of an arrangement $\mathcal{A}$ is the characteristic polynomial $\chi(\mathcal{A}, t) \in \mathbb{Z}[t]$. Indeed the characteristic polynomial is related to several other invariants, such as the Poincaré polynomial of the complexified complement $M(\mathcal{A})$ [19], the number of chambers for real arrangements [31], the number of $\mathbb{F}_{q}$-rational points $[10,26]$, Chern classes of certain vector bundles $[18,1]$, and lattice points countings $[7,14,15,16,30]$.

\subsection{Main results}

Let $V=\mathbb{R}^{\ell}$ be an $\ell$-dimensional Euclidean space. Let $\Phi \subset V^{*}$ be an irreducible root system. Fix a positive system $\Phi^{+} \subset \Phi$. For a positive root $\alpha \in \Phi^{+}$and $k \in \mathbb{Z}$, define

$$
H_{\alpha, k}=\{x \in V \mid \alpha(x)=k\} .
$$

The set of all such hyperplanes is called the affine Weyl arrangement. Finite truncations of the affine Weyl arrangement have received considerable attention ([2, 3, 4, 5, 11, 21, 22, 27, 29]). Among others, the (extended) Linial arrangement $\mathcal{L}_{\Phi}^{m}$ is defined by

$$
\mathcal{L}_{\Phi}^{m}=\left\{H_{\alpha, k} \mid \alpha \in \Phi^{+}, k=1,2, \ldots, m\right\},
$$

(where $\mathcal{L}_{\Phi}^{0}=\emptyset$ by convention). In [21], Postnikov and Stanley studied combinatorial aspects of Linial arrangements. They posed the following conjecture. 
Conjecture 1.1. ([21, Conjecture 9.14]) Suppose $m \geq 1$. Then every root $\alpha \in \mathbb{C}$ of the equation $\chi\left(\mathcal{L}_{\Phi}^{m}, t\right)=0$ satisfies $\operatorname{Re} \alpha=\frac{m h}{2}$, where $h$ denotes the Coxeter number of $\Phi$.

The conjecture was verified for $\Phi=A_{\ell}$ by Postnikov and Stanley [21], and for $\Phi=B_{\ell}, C_{\ell}$, and $D_{\ell}$ by Athanasiadis ([4]). These works are based on explicit representations of $\chi\left(\mathcal{L}_{\Phi}^{m}, t\right)$ for the corresponding root systems. (The case $\Phi=G_{2}$ is also easy.)

For exceptional root systems, some partial answers have recently been reported in [30]. Namely, for $\Phi \in\left\{E_{6}, E_{7}, E_{8}, F_{4}\right\}$, Conjecture 1.1 has been verified when the parameter $m>0$ satisfies

$$
m \equiv-1 \begin{cases}\bmod 6, & \Phi=E_{6}, E_{7}, F_{4}, \\ \bmod 30, & \Phi=E_{8}\end{cases}
$$

The purpose of this paper is to prove Conjecture 1.1 for exceptional root systems when $m \gg 0$. The main result is the following.

Theorem 1.2. (Corollary 5.14) Let $\Phi \in\left\{E_{6}, E_{7}, E_{8}, F_{4}\right\}$. Suppose $m \gg 0$. Then, every root $\alpha \in \mathbb{C}$ of the equation $\chi\left(\mathcal{L}_{\Phi}^{m}, t\right)=0$ satisfies $\operatorname{Re} \alpha=\frac{m h}{2}$.

\subsection{What makes roots lie on a line?}

The proof of Theorem 1.2 relies on the expression of the characteristic quasipolynomial $\chi_{\text {quasi }}\left(\mathcal{L}_{\Phi}^{m}, q\right)$ in terms of the Ehrhart quasi-polynomials and Eulerian polynomials developed in [30]. (See §2.) However, the key result that enables us to conclude "having the same real part" is the following elementary lemma.

Lemma 1.3. Let $f(t) \in \mathbb{R}[t]$. Suppose $M$ is a real number that satisfies the inequality

$$
M>2 \cdot \max \{\operatorname{Re} z \mid z \in \mathbb{C}, f(z)=0\} .
$$

Let $\omega \in \mathbb{C}$ be a complex number with $|\omega|=1$. Then, any root $z$ of the equation

$$
f(t)-\omega \cdot f(M-t)=0
$$

satisfies $\operatorname{Re} z=\frac{M}{2}$.

Proof. Set $f(t)=a\left(t-\alpha_{1}\right)\left(t-\alpha_{2}\right) \cdots\left(t-\alpha_{n}\right),(a \neq 0)$. As $f(t)$ is a real polynomial, $\overline{\alpha_{i}}$ is also a root of $f(t)$. Set $\beta_{i}=M-\overline{\alpha_{i}}$. Then, $\alpha_{i}$ and $\beta_{i}$ are symmetric with respect to the line $\operatorname{Re} z=\frac{M}{2}$, and we have $f(M-t)=$ $(-1)^{n} \cdot a \cdot \prod_{i=1}^{n}\left(t-\beta_{i}\right)$. If $\operatorname{Re} z<\frac{M}{2}$, then $\left|z-\alpha_{i}\right|<\left|z-\beta_{i}\right|$ for all $i$ (note that $\left.\operatorname{Re} \alpha_{i}<\frac{M}{2}\right)$, and hence $|f(z)|<|f(M-z)|$. Similarly, $\operatorname{Re} z>\frac{M}{2}$ implies $|f(z)|>|f(M-z)|$. Therefore, $f(z)=\omega f(M-z)$ implies that $\operatorname{Re} z=\frac{M}{2}$. 
The basic strategy of the proof of Theorem 1.2 is to construct $F^{(m)}(t) \in$ $\mathbb{Q}[t]$ such that

$$
\chi\left(\mathcal{L}_{\Phi}^{m}, t\right)=F^{(m)}(t)+(-1)^{\ell} \cdot F^{(m)}(m h-t),
$$

where $\ell$ is the rank of $\Phi$, then apply Lemma 1.3.

The remainder of this paper is organized as follows. In $\S 2$, we recall the notion of the characteristic quasi-polynomial $\chi_{\text {quasi }}(\mathcal{A}, q)$ for an integral arrangement $\mathcal{A}$. The characteristic quasi-polynomial $\chi_{\text {quasi }}\left(\mathcal{L}_{\Phi}^{m}, q\right)$ of the Linial arrangement $\mathcal{L}_{\Phi}^{m}$ can be expressed in terms of the Ehrhart quasi-polynomial $L_{\Phi}(t)$ of the fundamental alcove and the Eulerian polynomial $R_{\Phi}(t)$ of $\Phi$. The relation between these objects is described as follows (Theorem 2.9)

$$
\chi_{\text {quasi }}\left(\mathcal{L}_{\Phi}^{m}, q\right)=R_{\Phi}\left(S^{m+1}\right) L_{\Phi}(q)
$$

where $S$ is the shift operator. In $\S 3$, using the symmetry of the Eulerian polynomial (Proposition 2.5), we introduce the truncated characteristic quasipolynomial $\chi_{\text {quasi }}^{1 / 2}\left(\mathcal{L}_{\Phi}^{m}, q\right)$, which satisfies

$$
\chi_{\text {quasi }}\left(\mathcal{L}_{\Phi}^{m}, q\right)=\chi_{\text {quasi }}^{1 / 2}\left(\mathcal{L}_{\Phi}^{m}, q\right)+(-1)^{\ell} \chi_{\text {quasi }}^{1 / 2}\left(\mathcal{L}_{\Phi}^{m}, m h-q\right),
$$

(Proposition 3.5). Since these functions are quasi-polynomials, it does not make sense to consider the roots. However, we will see that the limit

$$
F_{\Phi}(q):=R_{\Phi}^{1 / 2}(S) q^{\ell}=\lim _{m \rightarrow \infty} \frac{\chi_{\text {quasi }}^{1 / 2}\left(\mathcal{L}_{\Phi}^{m}, m q\right)}{m^{\ell}}
$$

becomes a polynomial in $q$ (Proposition 3.1). The location of the zeros of $F_{\Phi}(t)$ is crucial for $m \gg 0$. Indeed, we will check, case-by-case, that the real parts of the zeros are less than $\frac{h}{2}$ (Proposition 3.7).

Because the proof for the quasi-polynomials is complicated, we will first give a simplified "polynomial version" of the main result as a "toy-case" in $\S 4$.

In $\S 5.1$, we summarize those properties of quasi-polynomials and Eulerian polynomials that are necessary for the proof of the main result. This is mainly to simplify the notation. In $\S 5.2$, we present a weaker version of the main result for the asymptotic behavior of the real parts of roots. In $\S 5.3$, we prove the main result.

\section{Preliminaries}

\subsection{Quasi-polynomials with the $G C D$-property}

A map $F: \mathbb{Z} \longrightarrow \mathbb{C}$ is called a quasi-polynomial with a period $\rho>0$ if $F(q)$ can be expressed as a polynomial in $q$ that depends only on the residue class 
$q \bmod \rho$. In other words, there exist polynomials $f_{0}, f_{1}, \ldots, f_{\rho-1} \in \mathbb{C}[t]$ such that

$$
F(q)=f_{i}(q)
$$

if $q \equiv i \bmod \rho$. The polynomials $f_{0}, \ldots, f_{\rho-1}$ are called the constituents of $F$. The period $\rho$ is said to be the minimal period if $F$ does not have smaller periods than $\rho$. The quasi-polynomial $F$ can be expressed as

$$
F(q)=c_{0}(q) q^{d}+c_{1}(q) q^{d-1}+\cdots+c_{d}(q),
$$

where $c_{i}: \mathbb{Z} \longrightarrow \mathbb{C}$ is a periodic function with a period $\rho$ (i.e., $c_{i}(q+\rho)=c_{i}(q)$ for all $q \in \mathbb{Z}$ ).

We say that the quasi-polynomial $F$ has a constant leading term if $c_{0}(q)$ in (1) is a nonzero constant function. In this case, $d$ is called the degree of the quasi-polynomial $F$.

The quasi-polynomial $F$ is said to have the $G C D$-property if the constituents satisfy: $\operatorname{gcd}(i, \rho)=\operatorname{gcd}(j, \rho) \Longrightarrow f_{i}(t)=f_{j}(t)$.

Remark 2.1. In this paper, we distinguish the roles of the variables $q$ and $t$. The variable $q$ always runs through $\mathbb{Z}$ (or $\mathbb{Z}_{>0}$ ), whereas $t$ runs through $\mathbb{R}$ or $\mathbb{C}$. Under this convention, the variable of a quasi-polynomial should be $q$, and its constituents may have a variable $t$.

\subsection{Characteristic quasi-polynomials}

Let $\mathcal{A}=\left\{H_{1}, \ldots, H_{n}\right\}$ be an arrangement of affine hyperplanes in $\mathbb{R}^{n}$. Throughout this paper, we assume that the hyperplanes are defined over $\mathbb{Z}$. More precisely, there exists an integral linear equation

$$
\alpha_{i}\left(x_{1}, \ldots, x_{\ell}\right)=a_{i 1} x_{1}+\cdots+a_{i \ell} x_{\ell}+b_{i}
$$

$\left(a_{i j}, b_{i} \in \mathbb{Z}\right)$ that satisfies $H_{i}=\alpha_{i}^{-1}(0) \subset \mathbb{R}^{\ell}$. For an arrangement $\mathcal{A}$, we can associate the modulo $q>0$ complement:

$$
M_{q}(\mathcal{A})=(\mathbb{Z} / q \mathbb{Z})^{\ell} \backslash \bigcup_{i=1}^{n} \bar{H}_{i}
$$

where $\bar{H}_{i}=\left\{x \in(\mathbb{Z} / q \mathbb{Z})^{\ell} \mid \alpha_{i}(x) \equiv 0 \bmod q\right\}$.

The following theorem was given by Kamiya, Takemura and Terao.

Theorem 2.2. ([14, 15, 16]) \# $M_{q}(\mathcal{A})$ is a quasi-polynomial with the $G C D$ property for sufficiently large $q \gg 0$ such that all constituents are monic of degree $\ell$. 
We denote the quasi-polynomial by $\chi_{\text {quasi }}(\mathcal{A}, q)$, which is called the characteristic quasi-polynomial of $\mathcal{A}$. The characteristic quasi-polynomial has a constant leading term; hence, it is of the form

$$
\chi_{\text {quasi }}(\mathcal{A}, q)=q^{\ell}+c_{1}(q) \cdot q^{\ell-1}+\cdots+c_{\ell}(q),
$$

where $c_{i}: \mathbb{Z} \longrightarrow \mathbb{Z}, i=1, \ldots, \ell$ are periodic functions. It is also known that the prime constituent of $\chi_{\text {quasi }}(\mathcal{A}, q)$ is equal to the characteristic polynomial of $\mathcal{A}[2,4]$, i. e., the characteristic polynomial $\chi(\mathcal{A}, t)$ has the form

$$
\chi(\mathcal{A}, t)=t^{\ell}+c_{1}(1) \cdot t^{\ell-1}+\cdots+c_{\ell}(1) .
$$

\subsection{Eulerian polynomials for root systems}

We first recall the terminology of $[8,13]$.

Let $V=\mathbb{R}^{\ell}$ be the Euclidean space with inner product $(\cdot, \cdot)$. Let $\Phi \subset V$ be an irreducible root system with exponents $e_{1}, \ldots, e_{\ell}$, Coxeter number $h$, and Weyl group $W$. (See Table 1 for explicit values.) For any integer $k \in \mathbb{Z}$ and $\alpha \in \Phi^{+}$, the affine hyperplane $H_{\alpha, k}$ is defined by

$$
H_{\alpha, k}=\{x \in V \mid(\alpha, x)=k\} \text {. }
$$

Fix a positive system $\Phi^{+} \subset \Phi$ and the set of simple roots $\Delta=\left\{\alpha_{1}, \ldots, \alpha_{\ell}\right\} \subset$ $\Phi^{+}$. The highest root, denoted by $\widetilde{\alpha} \in \Phi^{+}$, can be expressed as a linear combination $\widetilde{\alpha}=\sum_{i=1}^{\ell} c_{i} \alpha_{i}\left(c_{i} \in \mathbb{Z}_{>0}\right)$. We also set $\alpha_{0}:=-\widetilde{\alpha}$ and $c_{0}:=1$. Then, we have the linear relation

$$
c_{0} \alpha_{0}+c_{1} \alpha+\cdots+c_{\ell} \alpha_{\ell}=0 .
$$

The coweight lattice $Z(\Phi)$ and the coroot lattice $\check{Q}(\Phi)$ are defined as

$$
\begin{aligned}
& Z(\Phi)=\left\{x \in V \mid\left(\alpha_{i}, x\right) \in \mathbb{Z}, \alpha_{i} \in \Delta\right\} \\
& \check{Q}(\Phi)=\sum_{\alpha \in \Phi} \mathbb{Z} \cdot \frac{2 \alpha}{(\alpha, \alpha)} .
\end{aligned}
$$

The coroot lattice $\check{Q}(\Phi)$ is a finite index subgroup of the coweight lattice $Z(\Phi)$. The index $\# \frac{Z(\Phi)}{\bar{Q}(\Phi)}=f$ is called the index of connection.

Let $\varpi_{i}^{\vee} \in Z(\Phi)$ be the dual basis of the simple roots $\alpha_{1}, \ldots, \alpha_{\ell}$, that is, $\left(\alpha_{i}, \varpi_{j}^{\vee}\right)=\delta_{i j}$. Then, $Z(\Phi)$ is a free abelian group generated by $\varpi_{1}^{\vee}, \ldots, \varpi_{\ell}^{\vee}$. We also have $c_{i}=\left(\varpi_{i}^{\vee}, \widetilde{\alpha}\right)$. 
Each connected component of $V \backslash \bigcup_{\substack{\alpha \in \Phi^{+} \\ k \in \mathbb{Z}}} H_{\alpha, k}$ is an open simplex, called an alcove. Define the fundamental alcove $\sigma_{\Phi}^{\circ}$ by

$$
\sigma_{\Phi}^{\circ}=\left\{\begin{array}{l|l}
x \in V & \begin{array}{l}
\left(\alpha_{i}, x\right)>0, \quad(1 \leq i \leq \ell) \\
\widetilde{\alpha}, x)<1
\end{array}
\end{array}\right\}
$$

The closure $\overline{\sigma_{\Phi}^{\circ}}=\left\{x \in V \mid\left(\alpha_{i}, x\right) \geq 0(1 \leq i \leq \ell),(\widetilde{\alpha}, x) \leq 1\right\}$ is a simplex with vertices $0, \frac{\varpi_{1}^{\vee}}{c_{1}}, \ldots, \frac{\varpi_{\ell}^{\vee}}{c_{\ell}} \in V$. The supporting hyperplanes of facets of $\overline{\sigma_{\Phi}^{\circ}}$ are $H_{\alpha_{1}, 0}, \ldots, H_{\alpha_{\ell}, 0}, H_{\widetilde{\alpha}, 1}$.

Using the linear relation (3), we define the function asc : $W \longrightarrow \mathbb{Z}$.

Definition 2.3. Let $w \in W$. Then, $\operatorname{asc}(w)$ is defined by

$$
\operatorname{asc}(w)=\sum_{\substack{0 \leq i \leq \ell \\ w\left(\alpha_{i}\right)>0}} c_{i} .
$$

Definition 2.4. The generalized Eulerian polynomial $R_{\Phi}(x)$ is defined by

$$
R_{\Phi}(x)=\frac{1}{f} \sum_{w \in W} x^{\operatorname{asc}(w)} .
$$

The following proposition gives some basic properties of $R_{\Phi}(x)$.

Proposition 2.5. ([17])

(1) $\operatorname{deg} R_{\Phi}(x)=h-1$.

(2) (Duality) $x^{h} \cdot R_{\Phi}\left(\frac{1}{x}\right)=R_{\Phi}(x)$.

(3) $R_{\Phi}(x) \in \mathbb{Z}[x]$.

(4) $R_{A_{\ell}}(x)$ is equal to the classical Eulerian polynomial. (See $[9,12,24]$ for classical Eulerian polynomials.)

The polynomial $R_{\Phi}(x)$ was introduced by Lam and Postnikov in [17]. They proved that $R_{\Phi}(x)$ can be expressed in terms of cyclotomic polynomials and classical Eulerian polynomials.

Theorem 2.6. ([17, Theorem 10.1]) Let $\Phi$ be an irreducible root system of rank $\ell$. Then,

$$
R_{\Phi}(x)=\left[c_{0}\right]_{x} \cdot\left[c_{1}\right]_{x} \cdot\left[c_{2}\right]_{x} \cdots\left[c_{\ell}\right]_{x} \cdot R_{A_{\ell}}(x),
$$

where $[c]_{x}=\frac{x^{c}-1}{x-1}$.

We will give an alternative proof of Theorem 2.6 in $§ 2.5$ using Ehrhart series. 


\subsection{Ehrhart quasi-polynomials for root systems}

It is known that the number of lattice points $L_{\Phi}(q)=\#\left(q \cdot \overline{\sigma_{\Phi}^{\circ}} \cap Z(\Phi)\right)$ in the dilate $q \cdot \overline{\sigma_{\Phi}^{\circ}}$ is a quasi-polynomial in $q$, called the Ehrhart quasi-polynomial of $\overline{\sigma_{\Phi}^{\circ}}$. (See $[6,24]$ for details on Ehrhart theory.) Suter [25] explicitly computed the Ehrhart quasi-polynomial $L_{\Phi}(q)$. Several useful conclusions may be summarized as follows.

Theorem 2.7. (Suter [25])

(i) The Ehrhart quasi-polynomial $L_{\Phi}(q)$ has the GCD-property.

(ii) $L_{\Phi}(q)$ has a leading coefficient which is the constant $\frac{f}{|W|}$.

(iii) The minimal period is $\widetilde{n}=\operatorname{lcm}\left(c_{1}, c_{2}, \ldots, c_{\ell}\right)$. (See Table 1 for explicit values.)

(iv) If $q \in \mathbb{Z}$ is relatively prime to the period $\widetilde{n}$, then

$$
L_{\Phi}(q)=\frac{f}{|W|}\left(q+e_{1}\right)\left(q+e_{2}\right) \cdots\left(q+e_{\ell}\right) .
$$

(v) $\operatorname{rad}(\widetilde{n}) \mid h$, where $\operatorname{rad}(\widetilde{n})=\prod_{\substack{p: p \text { rime } \\ p \mid \tilde{n}}} p$ is the radical of $\widetilde{n}$.

(vi) The Ehrhart series $\operatorname{Ehr}_{\Phi}(z)$ of $L_{\Phi}(q)$ is

$$
\operatorname{Ehr}_{\Phi}(z):=\sum_{q=0}^{\infty} L_{\Phi}(q) z^{q}=\frac{1}{\left(1-z^{c_{0}}\right)\left(1-z^{c_{1}}\right) \cdots\left(1-z^{c_{\ell}}\right)}
$$

\begin{tabular}{c|c|c|c|c|c|c|c}
$\Phi$ & $e_{1}, \ldots, e_{\ell}$ & $c_{1}, \ldots, c_{\ell}$ & $h$ & $f$ & $|W|$ & $\tilde{n}$ & $\operatorname{rad}(\widetilde{n})$ \\
\hline \hline$A_{\ell}$ & $1,2, \ldots, \ell$ & $1,1, \ldots, 1$ & $\ell+1$ & $\ell+1$ & $(\ell+1) !$ & 1 & 1 \\
$B_{\ell}, C_{\ell}$ & $1,3,5, \ldots, 2 \ell-1$ & $1,2,2, \ldots, 2$ & $2 \ell$ & 2 & $2^{\ell} \cdot \ell !$ & 2 & 2 \\
$D_{\ell}$ & $1,3,5, \ldots, 2 \ell-3, \ell-1$ & $1,1,1,2, \ldots, 2$ & $2 \ell-2$ & 4 & $2^{\ell-1} \cdot \ell !$ & 2 & 2 \\
$E_{6}$ & $1,4,5,7,8,11$ & $1,1,2,2,2,3$ & 12 & 3 & $2^{7} \cdot 3^{4} \cdot 5$ & 6 & 6 \\
$E_{7}$ & $1,5,7,9,11,13,17$ & $1,2,2,2,3,3,4$ & 18 & 2 & $2^{10} \cdot 3^{4} \cdot 5 \cdot 7$ & 12 & 6 \\
$E_{8}$ & $1,7,11,13,17,19,23,29$ & $2,2,3,3,4,4,5,6$ & 30 & 1 & $2^{14} \cdot 3^{5} \cdot 5^{2} \cdot 7$ & 60 & 30 \\
$F_{4}$ & $1,5,7,11$ & $2,2,3,4$ & 12 & 1 & $2^{7} \cdot 3^{2}$ & 12 & 6 \\
$G_{2}$ & 1,5 & 2,3 & 6 & 1 & $2^{2} \cdot 3$ & 6 & 6
\end{tabular}

Table 1: Table of root systems.

The following proposition follows from Ehrhart-Macdonald reciprocity.

\section{Proposition 2.8.}

$$
L_{\Phi}(-q)=(-1)^{\ell} \cdot L_{\Phi}(q-h) .
$$

(See [30, Corollary 3.4] for a more general formula.) 


\subsection{Characteristic quasi-polynomials of Linial arrange- ments}

Let $S$ be the shift operator that replaces the variable $q$ by $q-1$ (or $t$ by $t-1)$. More generally, the polynomial $\sigma(S)=a_{0}+a_{1} S+\cdots+a_{d} S^{d}$ acts on a function $f(q)$ as

$$
\sigma(S) f(q)=a_{0} f(q)+a_{1} f(q-1)+\cdots+a_{d} f(q-d) .
$$

The characteristic quasi-polynomial $\chi_{\text {quasi }}\left(\mathcal{L}_{\Phi}^{n}, q\right)$ can be expressed in terms of the Eulerian polynomial $R_{\Phi}(x)$, and the Ehrhart quasi-polynomial $L_{\Phi}(q)$ of the fundamental alcove.

Theorem 2.9. ([30])

$$
\chi_{\text {quasi }}\left(\mathcal{L}_{\Phi}^{m}, q\right)=R_{\Phi}\left(S^{m+1}\right) L_{\Phi}(q)
$$

Remark 2.10. The formula (7) holds for $m=0$ if we consider $\mathcal{L}_{\Phi}^{0}$ to be the empty arrangement. In that case, we have

$$
q^{\ell}=R_{\Phi}(S) L_{\Phi}(q)
$$

This can be considered as a root system generalization of the Worpitzky identity [28].

Note that Theorem 2.6 by Lam and Postnikov follows from the Worpitzky identity (8) and Theorem 2.7 (vi). Indeed, equation (8) is equivalent to

$$
R_{\Phi}(z) \operatorname{Ehr}_{\Phi}(z)=\sum_{k=0}^{\infty} k^{\ell} z^{k}
$$

Note that the right-hand side depends only on the rank $\ell$. A comparison of (9) with $\Phi=A_{\ell}$ shows that

$$
\frac{R_{\Phi}(z)}{\left(1-z^{c_{0}}\right)\left(1-z^{c_{1}}\right) \cdots\left(1-z^{c_{\ell}}\right)}=\frac{R_{A_{\ell}}(z)}{(1-z)^{\ell+1}} .
$$

This yields Theorem 2.6.

Example 2.11. Let $\Phi=G_{2}$. Since $R_{A_{2}}(x)=x+x^{2}$, we have

$$
R_{G_{2}}(x)=(1+x)\left(1+x+x^{2}\right)\left(x+x^{2}\right)=x+3 x^{2}+4 x^{3}+3 x^{4}+x^{5} .
$$


The closed fundamental alcove $\overline{\sigma_{\Phi}^{\circ}}$ is the convex hull of $0, \frac{\varpi_{1}^{\vee}}{2}, \frac{\varpi_{1}^{\vee}}{3}$. The period is $\widetilde{n}=6$. The Ehrhart quasi-polynomial is

$$
L_{G_{2}}(q)= \begin{cases}\frac{1}{12}(q+1)(q+5), & \text { if } q \equiv 1,5 \bmod 6, \\ \frac{1}{12}(q+2)(q+4), & \text { if } q \equiv 2,4 \bmod 6 \\ \frac{1}{12}(q+3)^{2}, & \text { if } q \equiv 3 \bmod 6 \\ \frac{1}{12}\left(q^{2}+6 q+12\right), & \text { if } q \equiv 0 \bmod 6 .\end{cases}
$$

It is known that the characteristic quasi-polynomial of the Weyl arrangement $\mathcal{A}_{\Phi}=\left\{H_{\alpha, 0} \mid \alpha \in \Phi^{+}\right\}$can be expressed as $\chi_{\text {quasi }}\left(\mathcal{A}_{\Phi}, q\right)=(-1)^{\ell} \frac{\# W}{f} L_{\Phi}(-q)$ $[5,16]$. Thus, we have

$$
\chi_{\text {quasi }}\left(\mathcal{A}_{G_{2}}, q\right)= \begin{cases}(q-1)(q-5), & \text { if } t \equiv 1,5 \bmod 6, \\ (q-2)(q-4), & \text { if } t \equiv 2,4 \bmod 6, \\ (q-3)^{2}, & \text { if } t \equiv 3 \bmod 6, \\ q^{2}-6 q+12, & \text { if } t \equiv 0 \bmod 6 .\end{cases}
$$

The characteristic quasi-polynomial of the Linial arrangement is

$$
\chi_{\text {quasi }}\left(\mathcal{L}_{G_{2}}^{1}, q\right)=\left\{\begin{array}{lll}
q^{2}-6 q+11 & q \equiv 1 \quad \bmod 2 \\
q^{2}-6 q+14 & q \equiv 0 & \bmod 2 .
\end{array}\right.
$$

\section{Limit Polynomials}

\subsection{Normalized limit polynomials}

Let $f(S) \in \mathbb{C}[S]$ be a polynomial of the shift operator $S$, and $g(t) \in \mathbb{C}[t]$. Assume $\operatorname{deg} g(t)=\ell$. Let us consider the polynomial

$$
g_{m}(t):=f\left(S^{m+1}\right) g(t)
$$

for $m \geq 0$.

\section{Proposition 3.1.}

$$
\lim _{m \rightarrow \infty} \frac{g_{m}(m t)}{m^{\ell}}=f(S) t^{\ell}
$$


Proof. Write $f(S)=\sum_{k=0}^{N} a_{k} S^{k}$ and $g(t)=\sum_{i=0}^{\ell} c_{i} t^{\ell-i}\left(c_{0} \neq 0\right)$. Then, we have,

$$
g_{m}(t)=f\left(S^{m+1}\right) g(t)=\sum_{k=0}^{N} \sum_{i=0}^{\ell} a_{k} c_{i} \cdot(t-k(m+1))^{\ell-i}
$$

Hence,

$$
\begin{aligned}
\lim _{m \rightarrow \infty} \frac{g_{m}(m t)}{m^{\ell}} & =\lim _{m \rightarrow \infty} \frac{1}{m^{\ell}} \sum_{i=0}^{\ell} \sum_{k=0}^{N} a_{k} c_{i} \cdot(m t-k(m+1))^{\ell-i} \\
& =\lim _{m \rightarrow \infty} \sum_{i=0}^{\ell} \frac{1}{m^{i}} \sum_{k=0}^{N} a_{k} c_{i} \cdot\left(t-k \frac{m+1}{m}\right)^{\ell-i} \\
& =\sum_{k=0}^{N} a_{k} c_{0} \cdot(t-k)^{\ell} \\
& =f(S) t^{\ell}
\end{aligned}
$$

\subsection{Truncated Eulerian polynomials}

Suppose $R_{\Phi}(x)=\sum_{i=1}^{h-1} a_{i} x^{i}$. Define the truncated Eulerian polynomial $R_{\Phi}^{1 / 2}(t)$ by

$$
R_{\Phi}^{1 / 2}(x)= \begin{cases}\sum_{1 \leq i<\frac{h}{2}} a_{i} x^{i}, & \text { if } h \text { is odd } \\ \sum_{1 \leq i<\frac{h}{2}} a_{i} x^{i}+\frac{a_{h / 2}}{2} x^{h / 2}, & \text { if } h \text { is even }\end{cases}
$$

Example 3.2. Let $\Phi=G_{2}$. Then, $R_{\Phi}^{1 / 2}(x)=x+3 x^{2}+2 x^{3}$.

The following is straightforward from Proposition 2.5 (2).

Proposition 3.3. $R_{\Phi}(x)=R_{\Phi}^{1 / 2}(x)+x^{h} \cdot R_{\Phi}^{1 / 2}\left(x^{-1}\right)$.

Using the truncated Eulerian polynomial $R_{\Phi}^{1 / 2}(x)$, we define the half characteristic quasi-polynomial $\chi_{\text {quasi }}^{1 / 2}\left(\mathcal{L}_{\Phi}^{m}, q\right)$ as follows.

$$
\chi_{\text {quasi }}^{1 / 2}\left(\mathcal{L}_{\Phi}^{m}, q\right):=R_{\Phi}^{1 / 2}\left(S^{m+1}\right) L_{\Phi}(q) .
$$

Note that $\chi_{\text {quasi }}^{1 / 2}\left(\mathcal{L}_{\Phi}^{m}, q\right)$ is a quasi-polynomial of the period $\widetilde{n}$, however, it does not have the $G C D$-property. 
Example 3.4. For $\Phi=G_{2}$, we have

$$
R_{G_{2}}^{1 / 2}(S) L_{G_{2}}(q)=\left\{\begin{array}{lll}
\frac{6 q^{2}+10 q}{12} & q \equiv 0 & \bmod 3 \\
\frac{6 q^{2}+10 q-4}{12} & q \equiv 1 & \bmod 3 \\
\frac{6 q^{2}+10 q+4}{12} & q \equiv 2 & \bmod 3
\end{array}\right.
$$

and

$$
\chi_{\text {quasi }}^{1 / 2}\left(\mathcal{L}_{G_{2}}^{1}, q\right)=R_{G_{2}}^{1 / 2}\left(S^{2}\right) L_{G_{2}}(q)=\left\{\begin{array}{lll}
\frac{3 q^{2}-8 q+12}{6} & q \equiv 0 & \bmod 6, \\
\frac{3 q^{2}-8 q+5}{6} & q \equiv 1 & \bmod 6, \\
\frac{3 q^{2}-8 q+10}{6} & q \equiv 2 & \bmod 6, \\
\frac{3 q^{2}-8 q+3}{6} & q \equiv 3 & \bmod 6, \\
\frac{3 q^{2}-8 q+14}{6} & q \equiv 4 & \bmod 6, \\
\frac{3 q^{2}-8 q+1}{6} & q \equiv 5 & \bmod 6 .
\end{array}\right.
$$

Proposition 3.5. The half characteristic quasi-polynomial $\chi_{\text {quasi }}^{1 / 2}\left(\mathcal{L}_{\Phi}^{m}, q\right)$ satisfies the following.

$$
\chi_{\text {quasi }}\left(\mathcal{L}_{\Phi}^{m}, q\right)=\chi_{\text {quasi }}^{1 / 2}\left(\mathcal{L}_{\Phi}^{m}, q\right)+(-1)^{\ell} \chi_{\text {quasi }}^{1 / 2}\left(\mathcal{L}_{\Phi}^{m}, m h-q\right) .
$$

Proof. Write $R_{\Phi}^{1 / 2}(x)=\sum_{i=1}^{\lfloor h / 2\rfloor} a_{i}^{\prime} x^{i}$. From Theorem 2.9 and Proposition 3.3, it follows that

$$
\chi_{\text {quasi }}\left(\mathcal{L}_{\Phi}^{m}, q\right)=\left(R_{\Phi}^{1 / 2}\left(S^{m+1}\right) L_{\Phi}\right)(q)+\left(S^{h(m+1)} R_{\Phi}^{1 / 2}\left(S^{-m-1}\right) L_{\Phi}\right)(q) .
$$

The first term on the right hand side is equal to $\chi_{\text {quasi }}^{1 / 2}\left(\mathcal{L}_{\Phi}^{m}, q\right)$ in $(13)$. We shall prove that the second term is equal to $(-1)^{\ell} \chi_{\text {quasi }}^{1 / 2}\left(\mathcal{L}_{\Phi}^{m}, m h-q\right)$. Indeed, using Proposition 2.8, we have

$$
\begin{aligned}
\left(S^{h(m+1)} R_{\Phi}^{1 / 2}\left(S^{-m-1}\right) L_{\Phi}\right)(q) & =\sum_{i=1}^{\lfloor h / 2\rfloor} a_{i}^{\prime} S^{h(m+1)-(m+1) i} L_{\Phi}(q) \\
& =\sum_{i=1}^{\lfloor h / 2\rfloor} a_{i}^{\prime} L_{\Phi}(q-h(m+1)+(m+1) i) \\
& =(-1)^{\ell} \sum_{i=1}^{\lfloor h / 2\rfloor} a_{i}^{\prime} L_{\Phi}(-q+h m-(m+1) i) \\
& =(-1)^{\ell} \sum_{i=1}^{\lfloor h / 2\rfloor} a_{i}^{\prime} S^{(m+1) i} L_{\Phi}(-q+h m) \\
& =(-1)^{\ell} \chi_{\text {quasi }}^{1 / 2}\left(\mathcal{L}_{\Phi}^{m}, m h-q\right) .
\end{aligned}
$$


The next corollary follows immediately by replacing $q$ by $m h-q$ in Proposition 3.5.

Corollary 3.6. $\chi_{\text {quasi }}\left(\mathcal{L}_{\Phi}^{m}, q\right)=(-1)^{\ell} \chi_{\text {quasi }}\left(\mathcal{L}_{\Phi}^{m}, m h-q\right)$.

Consider $F_{\Phi}(t):=R_{\Phi}^{1 / 2}(S) t^{\ell}=\sum_{i=1}^{\lfloor h / 2\rfloor} a_{i}^{\prime}(t-i)^{\ell}$ as a polynomial in $t$. The distribution of the roots of $F_{\Phi}(t)=0$ will play a crucial role.

Proposition 3.7. Let $\Phi \in\left\{E_{6}, E_{7}, E_{8}, F_{4}, G_{2}\right\}$ be an exceptional root system. Suppose $F_{\Phi}(\alpha)=0, \alpha \in \mathbb{C}$. Then, $\operatorname{Re} \alpha<\frac{h}{2}$.

Proof. This proposition can be verified computationally. We describe the method for the case $\Phi=E_{6}$. The other cases are similar. First, using Table 1, Theorem 2.6, and $R_{A_{6}}(x)=x+57 x^{2}+302 x^{3}+302 x^{4}+57 x^{5}+x^{6}$, we have

$$
\begin{aligned}
R_{E_{6}}(x)= & (1+x)^{3}\left(1+x+x^{2}\right)\left(x+57 x^{2}+302 x^{3}+302 x^{4}+57 x^{5}+x^{6}\right) \\
= & x+61 x^{2}+537 x^{3}+1916 x^{4}+3782 x^{5}+4686 x^{6}+3782 x^{7} \\
& +1916 x^{8}+537 x^{9}+61 x^{10}+x^{11} .
\end{aligned}
$$

Therefore, we have

$$
R_{E_{6}}^{1 / 2}(x)=x+61 x^{2}+537 x^{3}+1916 x^{4}+3782 x^{5}+2343 x^{6}
$$

and

$R_{E_{6}}^{1 / 2}(S) t^{6}=(t-1)^{6}+61(t-2)^{6}+537(t-3)^{6}+1916(t-4)^{6}+3782(t-5)^{6}+2343(t-6)^{6}$.

The roots of $R_{E_{6}}^{1 / 2}(S) t^{6}=0$ (approximation by Mathematica) are $t=4.55334 \pm$ $0.465487 \sqrt{-1}, 4.78675 \pm 1.55735 \sqrt{-1}$, and $5.37033 \pm 3.11072 \sqrt{-1}$. All roots have real parts that are less than $\frac{h}{2}=6$.

The maximum real parts of the roots are presented in Table 2.

\begin{tabular}{c|c|c}
$\Phi$ & $\max \{\operatorname{Re} \alpha\}$ & $h / 2$ \\
\hline \hline$E_{6}$ & 5.3703 & 6 \\
$E_{7}$ & 8.4367 & 9 \\
$E_{8}$ & 14.6604 & 15 \\
$F_{4}$ & 4.8967 & 6 \\
$G_{2}$ & 2.166 & 3
\end{tabular}

Table 2: The maximal real parts of roots. 


\section{A Toy Case}

Let $\Phi \in\left\{E_{6}, E_{7}, E_{8}, F_{4}, G_{2}\right\}$. Denote its exponents by $e_{1}, \ldots, e_{\ell}$ and the Coxeter number by $h$. Let $g(t) \in \mathbb{R}[t]$ be a real polynomial of $\operatorname{deg} g=\ell$ that satisfies $g(t-h)=(-1)^{\ell} g(-t)$. (Note that such a polynomial exists, e. g., $g(t)=\prod_{i=1}^{\ell}\left(t+e_{i}\right)$.)

Theorem 4.1. With the above notation, for sufficiently large $m \gg 0$, every root $\alpha \in \mathbb{C}$ of the equation $\left(R_{\Phi}\left(S^{m+1}\right) g\right)(t)=0$ satisfies $\operatorname{Re} \alpha=\frac{m h}{2}$.

Proof. Set $g_{m}(t):=\left(R_{\Phi}^{1 / 2}\left(S^{m+1}\right) g\right)(t)$. Then, we have

$$
\left(R_{\Phi}\left(S^{m+1}\right) g\right)(t)=g_{m}(t)+(-1)^{\ell} g_{m}(m h-t) .
$$

(The proof is similar to that of Proposition 3.5.) By Proposition 3.1,

$$
\lim _{m \rightarrow \infty} \frac{g_{m}(m t)}{m^{\ell}}=R_{\Phi}^{1 / 2}(S) t^{\ell}=: F_{\Phi}(t) .
$$

From Proposition 3.7, it follows that the real parts of the roots of the equation $F_{\Phi}(t)=0$ are less than $\frac{h}{2}$. By the continuity of the roots of polynomials, for sufficiently large $m \gg 0$, the real parts of the roots of $g_{m}(m t)=0$ are also less than $\frac{h}{2}$, which is equivalent to saying that the real parts of the roots of $g_{m}(t)=0$ are less than $\frac{m h}{2}$. Then Lemma 1.3 completes the proof.

\section{$5 \quad$ Main Results}

In this section, by generalizing the argument in $\S 4$, we will prove the main result. The main difficulty is related to the fact that $L_{\Phi}(q)$ is a quasipolynomial. We will use the idea of averaging constituents to resolve this problem. We will work in the generalized setting described in $\S 5.1$ for the sake of notational simplicity.

\section{$5.1 \quad$ Settings}

Let $\widetilde{n}, h>0$ be positive integers and $L(q)$ be a quasi-polynomial with period $\tilde{n}$.

Assumption 5.1. $L(q)$ has a constant leading term of degree $\ell>0$. In other words, $L(q)$ has an expression of the form

$$
L(q)=c_{0} q^{\ell}+c_{1}(q) q^{\ell-1}+\cdots+c_{\ell}(q),
$$

where $c_{i}: \mathbb{Z} \longrightarrow \mathbb{Q}(i=1, \ldots, \ell)$ is a periodic function and $c_{0}$ is a nonzero constant. 
Assumption 5.2. $L(q)$ satisfies the following.

$$
L(-q)=(-1)^{\ell} L(q-h)
$$

Let $R(x) \in \mathbb{Q}[x]$ be a polynomial of $\operatorname{deg} R(x)=h-1$.

Assumption 5.3. $R(x)$ satisfies the following.

$$
x^{h} R\left(x^{-1}\right)=R(x) .
$$

Write $R(x)=a_{1} x+a_{2} x^{2}+\cdots+a_{h-1} x^{h-1}$. Define the truncation $R^{\prime}(x)$ of $R(x)$ by

$$
R^{\prime}(x)= \begin{cases}\sum_{1 \leq i<\frac{h}{2}} a_{i} x^{i}, & \text { if } h \text { is odd } \\ \sum_{1 \leq i<\frac{h}{2}} a_{i} x^{i}+\frac{a_{h / 2}}{2} x^{h / 2}, & \text { if } h \text { is even }\end{cases}
$$

We also write $R^{\prime}(x)=\sum_{i=1}^{\lfloor h / 2\rfloor} a_{i}^{\prime} x^{i}$. It is easy to see that $R(x)$ satisfies

$$
R(x)=R^{\prime}(x)+x^{h} R^{\prime}\left(x^{-1}\right) .
$$

Next, we make an assumption on the location of the roots of the polynomial

$$
R^{\prime}(S) t^{\ell}=\sum_{i=1}^{\lfloor h / 2\rfloor} a_{i}^{\prime}(t-i)^{\ell} .
$$

Assumption 5.4. Every root $\alpha \in \mathbb{C}$ of $R^{\prime}(S) t^{\ell}=0$ satisfies

$$
\operatorname{Re} \alpha<\frac{h}{2} \text {. }
$$

The following is our main example.

Example 5.5. Let $\Phi \in\left\{E_{6}, E_{7}, E_{8}, F_{4}, G_{2}\right\}$. Then, the Ehrhart quasipolynomial $L_{\Phi}(q)$, period $\widetilde{n}$, Coxeter number $h$, and Eulerian polynomial $R_{\Phi}(x)$ satisfy Assumptions 5.1, 5.2, 5.3, and 5.4.

Remark 5.6. Although Example 5.5 is the main example, we can construct many other examples that satisfy the above assumptions. For instance, for $\widetilde{n}=1$, some arbitrary $h \geq 3$ and $\ell>0, L(q)=\left(q+\frac{h}{2}\right)^{\ell}$ and $R(x)=x+x^{h-1}$ satisfy the above assumptions. 


\subsection{Asymptotic behavior of roots}

For $m>0$, define the quasi-polynomials $L^{(m)}(q)$ and $L^{\prime(m)}(q)$ of period $\tilde{n}$ by

$$
L^{(m)}(q)=\left(R\left(S^{m+1}\right) L\right)(q)
$$

and

$$
L^{\prime(m)}(q)=\left(R^{\prime}\left(S^{m+1}\right) L\right)(q) .
$$

By Assumption 5.2 (and Assumption 5.3),

$$
L^{(m)}(q)=L^{\prime(m)}(q)+(-1)^{\ell} L^{\prime(m)}(m h-q) .
$$

Let us denote the constituents by $L_{d}^{(m)}(t) \in \mathbb{Q}[t]$ for each residue class $d$ $\bmod \widetilde{n}($ or $0 \leq d<\widetilde{n})$, namely,

$$
L^{(m)}(q)=L_{d}^{(m)}(q) \text {, when } q \equiv d \bmod \widetilde{n} .
$$

Note that the constituents have the following expression:

$$
L_{d}^{(m)}(t)=\sum_{i=1}^{h-1} a_{i} \sum_{j=0}^{\ell} c_{j}(d-(m+1) i) \cdot(t-(m+1) i)^{\ell-j} .
$$

The relation (21) can also be written as

$$
L_{d}^{(m)}(t)=L_{d}^{\prime(m)}(t)+(-1)^{\ell} \cdot L_{m h-d}^{\prime(m)}(m h-t)
$$

at the level of the constituents.

\section{Proposition 5.7.}

$$
\lim _{m \rightarrow \infty} \frac{L_{d}^{\prime(m)}(m t)}{m^{\ell}}=c_{0} \cdot R^{\prime}(S) t^{\ell}
$$

In particular, the limit does not depend on the residue $d$.

Proof. Recall $L_{d}^{\prime(m)}(t)=\sum_{i=1}^{\lfloor h / 2\rfloor} a_{i}^{\prime} \sum_{j=0}^{\ell} c_{j}(d-(m+1) i)(t-(m+1) i)^{\ell-j}$. Hence,

$$
\frac{L_{d}^{\prime(m)}(m t)}{m^{\ell}}=\sum_{i=1}^{\lfloor h / 2\rfloor} a_{i}^{\prime} \sum_{j=0}^{\ell} \frac{c_{j}(d-(m+1) i)}{m^{j}}\left(t-\frac{m+1}{m} i\right)^{\ell-j} .
$$

When $j>0$, since $c_{j}$ is a periodic function, we have $\lim _{m \rightarrow \infty} \frac{c_{j}(d-(m+1) i)}{m^{j}}=0$. By the assumption, $c_{0}(d-(m+1) i)=c_{0}$ is a nonzero constant. We have

$$
\begin{aligned}
\lim _{m \rightarrow \infty} \frac{L_{d}^{\prime(m)}(m t)}{m^{\ell}} & =c_{0} \cdot \sum_{i=1}^{\lfloor h / 2\rfloor} a_{i}^{\prime}(t-i)^{\ell} \\
& =c_{0} \cdot R^{\prime}(S) t^{\ell} .
\end{aligned}
$$




\section{Definition 5.8.}

$$
\begin{aligned}
\bar{r}_{d}^{(m)} & :=\max \left\{\operatorname{Re} \alpha \mid \alpha \in \mathbb{C}, L_{d}^{(m)}(\alpha)=0\right\}, \\
\underline{r}_{d}^{(m)} & :=\min \left\{\operatorname{Re} \alpha \mid \alpha \in \mathbb{C}, L_{d}^{(m)}(\alpha)=0\right\} .
\end{aligned}
$$

If $L(q)$ and $R(x)$ satisfy Assumptions 5.1-5.4, then $\bar{r}_{d}^{(m)}$ and $\underline{r}_{d}^{(m)}$ approach $\frac{m h}{2}$ as $m \rightarrow \infty$. More precisely, we have the following.

Theorem 5.9. For any $0 \leq d<\tilde{n}$,

$$
\lim _{m \rightarrow \infty} \frac{\bar{r}_{d}^{(m)}}{m}=\lim _{m \rightarrow \infty} \frac{\underline{r}_{d}^{(m)}}{m}=\frac{h}{2} .
$$

Proof. Let $F(t):=c_{0} \cdot R^{\prime}(S) t^{\ell}$. Then, by (22) and Proposition 5.7, we have

$$
\lim _{m \rightarrow \infty} \frac{L_{d}^{(m)}(m t)}{m^{\ell}}=F(t)+(-1)^{\ell} \cdot F(h-t) .
$$

Choose a root $\alpha_{m} \in \mathbb{C}$ of $L_{d}^{(m)}(t)=0$. Then, obviously, $\frac{\alpha_{m}}{m}$ satisfies the equation $L_{d}^{(m)}(m t)=0$. Hence, $\frac{\alpha_{m}}{m}$ approaches the (set of) roots of $F(t)+$ $(-1)^{\ell} F(h-t)=0$. Equation (23) follows from Assumption 5.4 and Lemma 1.3 .

Corollary 5.10. Let $\Phi \in\left\{E_{6}, E_{7}, E_{8}, F_{4}\right\}$. Fix $0 \leq d<\widetilde{n}$. Let $\alpha_{m} \in \mathbb{C}$ be a root of the constituent $\chi_{\mathrm{quasi}, d}\left(\mathcal{L}_{\Phi}^{m}, t\right)$. Then,

$$
\lim _{m \rightarrow \infty} \frac{\operatorname{Re} \alpha_{m}}{m}=\frac{h}{2}
$$

\subsection{Exact arrangement of roots}

In the previous subsection, we proved that the real part of a root $\alpha$ of a constituent $L_{d}^{(m)}(t)=0$ is asymptotically close to $\frac{m h}{2}$. Here, we prove a stronger result for some special constituents.

Definition 5.11. (Using the notation in $\S 5.1)$, the residue $d \bmod \widetilde{n}(0 \leq$ $d<\widetilde{n}$ ) is called an admissible residue if the constituents of $L^{(m)}(q)$ satisfy

$$
L_{d}^{(m)}(t)=L_{d+k h}^{(m)}(t)=L_{-d+k h}^{(m)}(t)
$$

for all $k \in \mathbb{Z}$ and $m>0$. 
Example 5.12. Consider the situation in Example 5.5. Then, $L^{(m)}(q)=$ $\left(R\left(S^{m+1}\right) L\right)(q)$ is equal to $\chi_{\text {quasi }}\left(\mathcal{L}_{\Phi}^{m}, q\right)$, which has the $G C D$-property. Hence, $d$ is an admissible residue if and only if

$$
\operatorname{gcd}(d, \widetilde{n})=\operatorname{gcd}(d+k h, \widetilde{n})
$$

for all $k \in \mathbb{Z}$. As $\operatorname{rad}(\widetilde{n}) \mid h$ (Theorem $2.7(\mathrm{v})$ ), $d=1$ is an admissible residue. Other admissible residues (divisors of $\widetilde{n}$ ) for $\Phi \in\left\{E_{6}, E_{7}, E_{8}, F_{4}, G_{2}\right\}$ are listed in Table 3.

\begin{tabular}{c|c|c|c|c|c}
$\Phi$ & $\tilde{n}$ & $\operatorname{rad}(\widetilde{n})$ & $h$ & admissible divisor of $\widetilde{n}$ & $m_{0}$ \\
\hline \hline$E_{6}$ & 6 & 6 & 12 & $1,2,3,6$ & 1 \\
$E_{7}$ & 12 & 6 & 18 & 1,3 & 2 \\
$E_{8}$ & 60 & 30 & 30 & $1,3,5,15$ & 2 \\
$F_{4}$ & 12 & 6 & 12 & $1,2,3,4,6,12$ & 1 \\
$G_{2}$ & 6 & 6 & 6 & $1,2,3,6$ & 1
\end{tabular}

Table 3: Admissible divisors.

The following is the main result.

Theorem 5.13. (Using the same notation as in §5.1.) Suppose $d$ ( $0 \leq d<$ $\widetilde{n})$ is an admissible residue. Let $\alpha_{m}$ be a root of $L_{d}^{(m)}(t)=0$. Then, for sufficiently large $m \gg 0$, Re $\alpha_{m}=\frac{m h}{2}$ holds.

Proof. Let $m_{0}:=\frac{\widetilde{n}}{\operatorname{gcd}(h, \widetilde{n})}$. Then, $L_{d}^{(m)}(t)=L_{d+k h}^{(m)}(t)=L_{-d+k h}^{(m)}(t)$ for $k=$ $0,1, \ldots, m_{0}-1$. Hence, using (22),

$$
\begin{aligned}
L_{d}^{(m)}(t) & =\frac{1}{m_{0}} \cdot \sum_{k=0}^{m_{0}-1} L_{d+k h}^{(m)}(t) \\
& =\frac{1}{m_{0}} \cdot \sum_{k=0}^{m_{0}-1} L_{d+k h}^{\prime(m)}(t)+\frac{(-1)^{\ell}}{m_{0}} \cdot \sum_{k=0}^{m_{0}-1} L_{-d+(m-k) h}^{\prime(m)}(m h-t) \\
& =\frac{1}{m_{0}} \cdot \sum_{k=0}^{m_{0}-1} L_{d+k h}^{\prime(m)}(t)+\frac{(-1)^{\ell}}{m_{0}} \cdot \sum_{k=0}^{m_{0}-1} L_{-d+k h}^{\prime(m)}(m h-t) .
\end{aligned}
$$

As $L_{d}^{(m)}(t)=L_{-d}^{(m)}(t)$, we also have

$$
L_{d}^{(m)}(t)=\frac{1}{m_{0}} \cdot \sum_{k=0}^{m_{0}-1} L_{-d+k h}^{\prime(m)}(t)+\frac{(-1)^{\ell}}{m_{0}} \cdot \sum_{k=0}^{m_{0}-1} L_{d+k h}^{\prime(m)}(m h-t) .
$$


Define the polynomial $F_{d}^{(m)}(t)$ by

$$
F_{d}^{(m)}(t):=\frac{1}{2 m_{0}} \cdot \sum_{k=0}^{m_{0}-1}\left\{L_{d+k h}^{\prime(m)}(t)+L_{-d+k h}^{\prime(m)}(t)\right\} .
$$

Then, combining $(26)$ and $(27), L_{d}^{(m)}(t)$ can be expressed as

$$
L_{d}^{(m)}(t)=F_{d}^{(m)}(t)+(-1)^{\ell} \cdot F_{d}^{(m)}(m h-t) .
$$

From Proposition 5.7, it follows that

$$
\lim _{m \rightarrow \infty} \frac{F_{d}^{(m)}(m t)}{m^{\ell}}=c_{0} \cdot R^{\prime}(S) t^{\ell}
$$

Hence, for sufficiently large $m \gg 0$, every root $\alpha \in \mathbb{C}$ of $F_{d}^{(m)}(t)=0$ satisfies

$$
\operatorname{Re} \alpha<\frac{m h}{2} \text {. }
$$

Applying Lemma 1.3, every root of $L_{d}^{(m)}(t)=0$ has the real part $\frac{m h}{2}$.

Corollary 5.14. Let $\Phi \in\left\{E_{6}, E_{7}, E_{8}, F_{4}, G_{2}\right\}$. For sufficiently large $m \gg$ 0 , every root $\alpha \in \mathbb{C}$ of the characteristic polynomial $\chi\left(\mathcal{L}_{\Phi}^{m}, t\right)$ of the Linial arrangement $\mathcal{L}_{\Phi}^{m}$ satisfies $\operatorname{Re} \alpha=\frac{m h}{2}$.

Proof. Recall that the characteristic polynomial is the constituent of the characteristic quasi-polynomial corresponding to $d=1$. Since $d=1$ is an admissible residue, we can apply Theorem 5.13.

Acknowledgements. The author was partially supported by JSPS KAKENHI Grant Number 25400060, 15KK0144, and 16K13741. He would also like to thank referees for the helpful suggestions.

\section{References}

[1] P. Aluffi, Grothendieck classes and Chern classes of hyperplane arrangements. IMRN 2013, no. 8, 1873-1900.

[2] C. A. Athanasiadis, Characteristic polynomials of subspace arrangements and finite fields. Adv. Math. 122 (1996), no. 2, 193-233.

[3] C. A. Athanasiadis, Deformations of Coxeter hyperplane arrangements and their characteristic polynomials. Arrangements - Tokyo 1998, 1-26, Adv. Stud. Pure Math., 27, Kinokuniya, Tokyo, 2000. 
[4] C. A. Athanasiadis, Extended Linial hyperplane arrangements for root systems and a conjecture of Postnikov and Stanley. J. Algebraic Combin. 10 (1999), no. 3, 207-225.

[5] C. A. Athanasiadis, Generalized Catalan numbers, Weyl groups and arrangements of hyperplanes. Bull. London Math. Soc. 36 (2004), no. 3, 294-302.

[6] M. Beck and S. Robins, Computing the continuous discretely. Integerpoint enumeration in polyhedra. Undergraduate Texts in Mathematics. Springer, New York, 2007. xviii+226 pp.

[7] A. Blass and B. Sagan, Characteristic and Ehrhart polynomials. J. Algebraic Combin. 7 (1998), no. 2, 115-126.

[8] N. Bourbaki, Groupes et algèbres de Lie, Ch. 4-6, Hermann, Paris, 1968.

[9] L. Comtet, Advanced combinatorics. The art of finite and infinite expansions. Revised and enlarged edition. D. Reidel Publishing Co., Dordrecht, 1974. xi+343 pp.

[10] H. Crapo, G. -C. Rota, On the foundations of combinatorial theory: combinatorial geometries. MIT press, Cambridge (1970).

[11] P. H. Edelman and V. Reiner, Free arrangements and rhombic tilings. Discrete Comput. Geom. 15 (1996), no. 3, 307-340.

[12] D. Foata, Eulerian polynomials: from Euler's time to the present. The legacy of Alladi Ramakrishnan in the mathematical sciences, 253-273, Springer, New York, 2010.

[13] J. E. Humphreys, Reflection groups and Coxeter groups. Cambridge Studies in Advanced Mathematics, 29. Cambridge University Press, Cambridge, 1990. xii+204 pp.

[14] H. Kamiya, A. Takemura and H. Terao, Periodicity of hyperplane arrangements with integral coefficients modulo positive integers. J. Algebraic Combin. 27 (2008), no. 3, 317-330.

[15] H. Kamiya, A. Takemura and H. Terao, Periodicity of non-central integral arrangements modulo positive integers. Ann. Comb. 15 (2011), no. 3, 449-464. 
[16] H. Kamiya, A. Takemura and H. Terao, The characteristic quasipolynomials of the arrangements of root systems and mid-hyperplane arrangements. Arrangements, local systems and singularities, 177-190, Progr. Math., 283, Birkhäuser Verlag, Basel, 2010.

[17] T. Lam and A. Postnikov, Alcoved polytopes II. arXiv preprint arXiv:1202.4015 (2012).

[18] M. Mustaţă and H. Schenck, The module of logarithmic p-forms of a locally free arrangement, J. Algebra 241 (2001), 699-719.

[19] P. Orlik and L. Solomon, Combinatorics and topology of complements of hyperplanes. Invent. Math. 56 (1980), 167-189.

[20] P. Orlik and H. Terao, Arrangements of hyperplanes. Grundlehren der Mathematischen Wissenschaften, 300. Springer-Verlag, Berlin, 1992. xviii+325 pp.

[21] A. Postnikov and R. Stanley, Deformations of Coxeter hyperplane arrangements. J. Combin. Theory Ser. A 91 (2000), no. 1-2, 544-597.

[22] J. -Y. Shi, The Kazhdan-Lusztig cells in certain affine Weyl groups. Lecture Notes in Mathematics, 1179. Springer-Verlag, Berlin, 1986. x+307 pp.

[23] R. Stanley, An introduction to hyperplane arrangements. Geometric combinatorics, 389-496, IAS/Park City Math. Ser., 13, Amer. Math. Soc., Providence, RI, 2007.

[24] R. Stanley, Enumerative combinatorics. Volume 1. Second edition. Cambridge Studies in Advanced Mathematics, 49. Cambridge University Press, Cambridge, 2012. xiv+626 pp.

[25] R. Suter, The number of lattice points in alcoves and the exponents of the finite Weyl groups. Math. Comp. 67 (1998), no. 222, 751-758.

[26] H. Terao, The Jacobians and the discriminants of finite reflection groups. Tohoku Math. J. (2) 41 (1989), no. 2, 237-247.

[27] H. Terao, Multiderivations of Coxeter arrangements. Invent. Math. 148 (2002), no. 3, 659-674.

[28] J. Worpitzky, Studien über die Bernoullischen und Eulerischen Zahlen. J. reine angew. Math. 94 (1883), 203-232. 
[29] M. Yoshinaga, Characterization of a free arrangement and conjecture of Edelman and Reiner. Invent. Math. 157 (2004), no. 2, 449-454.

[30] M. Yoshinaga, Worpitzky partitions for root systems and characteristic quasi-polynomials. To appear in Tohoku Math. J.

[31] T. Zaslavsky, Facing up to arrangements: Face-count formulas for partitions of space by hyperplanes. Memoirs Amer. Math. Soc. 1 (1975), no. 154, vii+102 pp. 